\title{
When is Menzerath-Altmann law mathematically triv- ial? A new approach
}

\author{
Ramon Ferrer-i-Cancho ${ }^{1}$, Antoni Hernández-Fernández ${ }^{1,2}$, Jaume Baixeries ${ }^{1}$, Łukasz \\ Dębowski $^{3}$ and Ján Mačutek ${ }^{4}$ \\ ${ }^{1}$ Complexity \& Quantitative Linguistics Lab, LARCA Research Group, Departa- \\ ment de Llenguatges i Sistemes Informàtics, Universitat Politècnica de Catalunya, \\ Campus Nord, Edifici Omega Jordi Girona Salgado 1-3, 08034 Barcelona (Catalo- \\ nia), Spain \\ ${ }^{2}$ Departament de Lingüística General, Universitat de Barcelona, Gran Via de les \\ Corts Catalanes 585, 08007 Barcelona (Catalonia), Spain \\ ${ }^{3}$ Institute of Computer Science, Polish Academy of Sciences, ul. Jana Kazimierza \\ 5, 01-248 Warszawa, Poland \\ ${ }^{4}$ Department of Applied Mathematics and Statistics, Comenius University, Mlyn- \\ ska dolina, 84248 Bratislava, Slovakia
}

\begin{abstract}
Menzerath's law, the tendency of $Z$, the mean size of the parts, to decrease as $X$, the number of parts, increases is found in language, music and genomes. Recently, it has been argued that the presence of the law in genomes is an inevitable consequence of the fact that $Z=Y / X$, which would imply that $Z$ scales with $X$ as $Z \sim 1 / X$. That scaling is a very particular case of MenzerathAltmann law that has been rejected by means of a correlation test between $X$ and $Y$ in genomes, being $X$ the number of chromosomes of a species, $Y$ its genome size in bases and $Z$ the mean chromosome size. Here we review the statistical foundations of that test and consider three non-parametric tests based upon different correlation metrics and one parametric test to evaluate if $Z \sim 1 / X$ in genomes. The most powerful test is a new non-parametric based upon the correlation ratio, which is able to reject $Z \sim 1 / X$ in nine out of eleven taxonomic groups and detect a borderline group. Rather than a fact, $Z \sim 1 / X$ is a baseline that real genomes do not meet. The view of Menzerath-Altmann law as inevitable is seriously flawed.
\end{abstract}

Keywords: Menzerath-Altmann law - power-laws - genomes - Monte Carlo methods

Corresponding author: R. Ferrer-i-Cancho, rferrericancho@1si.upc.edu 


\section{Introduction}

Consider that $X$ and $Y$ are two discrete random variables and that $Z=Y / X$ with $X \neq 0$. For the particular case that $Z$ is a mean, Menzerath's law is the tendency of $Z$ to decrease as $X$ increases (Menzerath, 1954, Li, 2012): $X$ stands for the number of parts of the construct (e.g., the number of clauses of a sentence), $Y$ stands for the size of the whole in parts (e.g., the length in words of the sentence) and $Z$ stands for the mean size of the construct (e.g., the mean length of the clauses in words). The scaling of $Z$ with $X$ that Menzerath's law describes qualitatively is typically modelled by means of Menzerath-Altmann law (Altmann, 1980, Cramer, 2005), i.e.

$$
Z=a X^{b} e^{c X}
$$

where $a, b$ and $c$ are the parameters of the model. Menzerath's law has been found in language (Menzerath, 1954) and genomes (Ferrer-i-Cancho and Forns, 2009, Li, 2012) and also indirectly through a wide range of studies where MenzerathAltmann law is fitted to human language e.g., (Altmann, 1980, Teupenhayn and Altmann, 1984), and music (Boroda and Altmann, 1991) and genomes (Wilde and Schwibbe, 1989, Li, 2012) which yields parameters $a, b$ and $c$ that support a negative correlation between $Z$ and $X$ at least for sufficiently large $X$ (see Cramer (2005) for a review of parameter values). Note that in our definition of these laws, Menzerath's law is a light and model neutral law. In our view, the only constraint imposed by that law is that $Z$ and $X$ are negatively correlated (the dependency between $Z$ and $X$ might not be functional) while Menzerath-Altmann law is a strong assumption for two reasons: it assumes that the dependency between $Z$ and $X$ is functional and takes the form defined by Eq. 1 .

Recently, it has been argued that $Z=Y / X$ leads inevitably to a power-law of the form (Solé, 2010)

$$
Z=a X^{-1}
$$

that is Menzerath-Altmann law with $b=-1$ and $c=0$. If the argument was correct, Menzerath-Altmann law would be a trivial scaling law at least from a mathematical perspective. Being $X$ the number of chromosome of a species, $Y$ the genome size in bases of that species and $Z$ its mean chromosome size, agreement with Eq. 2 has been claimed simply by fitting Menzerath-Altmann law with $c=0$ and obtaining $b \approx 1$ (Solé, 2010). However, more than a decade of statistical research on presumable power laws in biology indicates that looks can be deceiving (May and Stumpf, 2000, Tanaka, Yi, and Doyle, 2005, Stumpf and Ingram, 2005, Khanin and Wit, 2006). Sometimes, the divergence of the degree distribution of biological networks from a power-law is obvious upon visual inspection if a convenient 
representation of the data is employed (Tanaka et al., 2005). In general, the hypothesis of a power-law for the degree distribution of biological networks has been rejected for different kinds of biological networks even when an exponentially truncated power-law similar to Eq. 1 1 was considered (Khanin and Wit, 2006). Modern model selection methods indicate that simple power-law models do not provide an adequate description of the degree distribution of protein interaction and metabolic networks (Stumpf and Ingram, 2005, Stumpf, Ingram, Nouvel, and Wiuf, 2005). The same has happened to other hypotetical power-laws after careful inspection (May and Stumpf, 2000, Tjørve, 2003). The dependency between the number of different species as a function of area is described more accurately by functions that are not power-laws (May and Stumpf, 2000). Interestingly, the power-law model (as well as the exponential model) is seen as lacking biological depth for describing the species-area relationship with regard to other possible functions (Tjørve, 2003). In a similar vein, Eq. 2 has been argued to imply assumptions that jeopardize chromosome well-formedness in the context of the relationship between chromosome number and mean chromosome length (Baixeries, Hernández-Fernández, and Ferrer-i-Cancho, 2012, Ferrer-i-Cancho, Forns, Hernández-Fernández, Bel-Enguix, and Baixeries, 2013b). This suggests that rather than indicators of complexity, pure power laws (or pure power-laws with a certain exponent, e.g., -1 in Eq. 2) might play the role of baselines in certain circumstances.

The main goal of this article is presenting a powerful and statistically rigorous methodological framework to test if a real sample follows the particular case of power-law defined by Eq. 2. Notice that the equation is a particular case of powerlaw for two reasons: the exponent of the power-law is -1 and, more importantly, the response variable $Z$ is $Z=Y / X$, being $X$ the predictor.

Whether Eq. 2holds in genomes for the relationship between chromosome number and mean chromosome size, has been debated (Solé, 2010, Ferrer-i-Cancho et al. 2013b, Hernández-Fernández, Baixeries, Forns, and Ferrer-i-Cancho, 2011, Baixeries et al., 2012, Ferrer-i-Cancho, Baixeries, and Hernández-Fernández, 2013a, Baixeries, Hernández-Fernández, Forns, and Ferrer-i-Cancho, 2013). This is part of a long-running debate on the depth and importance of statistical laws of language in science (e.g., Miller and Chomsky (1963), Li (1992), Suzuki, Tyack, and Buck (2005), McCowan, Doyle, Jenkins, and Hanser (2005), Solé (2010), Ferrer-iCancho et al. (2013b)). These laws are seen by many as inevitable (Miller, 1968 , Solé, 2010), useless (Suzuki et al., 2005) or lacking mechanistic sophistication (Li, 1992, Stumpf and Porter, 2012). Here we aim to contribute to this general debate from the perspective of Menzerath-Altmann law with new theoretical insights and new experiments on genomes. In particular, we will provide some theoretical foundations for testing if Eq. 2 holds. It will be shown that rejecting Eq. 2 if $X$ and $Y$ are correlated (Baixeries et al., 2012, Hernández-Fernández et al., 2011) is correct 
but conservative. Furthermore, a new test that rejects Eq. 2 in all taxonomic groups considered so far except two (one being a borderline case) will be presented. The view of Menzerath-Altmann law as inevitable (Solé, 2010) is seriously flawed.

\section{Statistical foundations}

\subsection{The meaning of $Z=a / X$}

According to standard modelling, claiming that $Z$ scales with $X$ following Eq. 2 can be recast as (Ritz and Streibig, 2008, pp. 1),

$$
E(Z \mid X=x)=a / x
$$

for any $x$, being $E(Z \mid X=x)$ the conditional expectation of $Z$ given $x$ (a concrete value of $X$ ). Testing if Eq. 2 holds reduces to testing if $Y$ is mean independent of $X$ (Poirier, 1995, pp. 67), namely $E(Y \mid X=x)=E(Y)$ for any $x$ (Ferrer-i-Cancho et al., 2013a). Formally, this is supported by the following theorem (Ferrer-i-Cancho et al., 2013a)

Theorem 2.1 Consider a constant $a$ and two random natural variables, $X$ and $Y$, and a third random number $Z$, such that $X>0$ and $Z=Y / X$. Then, $E(Z \mid X=x)=$ $a / x$ if and only if $Y$ is mean independent of $X$, i.e. $E(Y \mid X=x)=E(Y)$ for any $x$.

Therefore, one condition for a trivial Menzerath-Altmann law is that $Y$ is mean independent of $X$. Mean independence is well-known in econometrics (Cameron and Trivedi, 2009, Wooldridge, 2010). Another more obvious mathematically trivial version of the law occurs when $Z$ is mean independent of $X$, i.e.

$$
E(Z \mid X=x)=E(Z),
$$

which is equivalent to constant $E(Z \mid X=x)$ (Ferrer-i-Cancho et al., 2013a). The analysis of the correlation between $Z$ and $X$ in genomes discarded this mean constancy of $Z$ for nine out of eleven taxonomic groups (Ferrer-i-Cancho and Forns, 2009) and the result was confirmed with an updated dataset (Baixeries et al., 2012). Therefore, Menzerath-Altmann law as a model of $E(Z \mid X=x)$ has two trivial versions:

- $b=c=0: Z$ is mean independent of $X$.

- $b=-1$ and $c=0: Y$ is mean independent of $X$. 
Interestingly, $b$ lies between 0 and -1 when $c=0$ is assumed: e.g., $b=-0.27 \pm$ 0.11 in language, being $Z$ is the mean clause length in sentences and $X$ is the number of sentences (Teupenhayn and Altmann, 1984), and $b=-0.44 \pm 0.09$ in music, being $Z$ is the mean F-motif length in tones and $X$ is the number of F-motifs (Boroda and Altmann, 1991). In both cases, we report $b=\mu \pm \sigma$, where $b$ is the exponent of a sample while $\mu$ and $\sigma$ are, the mean and the standard deviation of $b$ in an ensemble of samples, respectively.

\subsection{Three definitions of lack of association between $X$ and $Y$}

For the remainder of sections, it is important to bear in mind the definition of three statistical relations between $X$ and $Y$ (Poirier, 1995, pp. 67-68):

- $X$ and $Y$ are independent: $p(Y=y \mid X=x)=p(Y=y)$ for any $x$ and $y$.

- $Y$ is mean independent of $X: E(Y \mid X=x)=E(Y)$ for any $x$.

- $X$ and $Y$ are Pearson uncorrelated: $\operatorname{COV}(X, Y)=0$ where $C O V(X, Y)=$ $E(X Y)-E(X) E(Y)$ is the covariance between $X$ and $Y$. Notice that Pearson uncorrelation, i.e. $\rho(X, Y)=0$, being $\rho(X, Y)$ the Pearson correlation coefficient, is equivalent to zero covariance as (DeGroot and Schervish, 2012)

$$
\rho(X, Y)=\frac{\operatorname{COV}(X, Y)}{\sigma(X) \sigma(Y)},
$$

with $\sigma(X)$ and $\sigma(Y)$ as the standard deviation of $X$ and $Y$, respectively.

As $X$ and $Y$ are Pearson uncorrelated if and only if $\rho(X, Y)=0$ (or $C O V(X, Y)=0$ ), $Y$ is mean independent of $X$ if and only if $\eta(Y, X)=0$, where $\eta(Y, X)$ is a lessknown association metric: the correlation ratio (Crathorne, 1922, Kruskal, 1958). $\eta(Y, X)$ derives from the variance of $E(Y \mid X=x)$, which is by definition,

$$
\operatorname{Var}(E(Y \mid X=x))=E\left([E(Y \mid X=x)-E(E(Y \mid X=x))]^{2}\right) .
$$

By the law of total probability for expectations (DeGroot and Schervish, 2012, pp. 258), $E(E(Y \mid X=x))=E(Y)$ and thus

$$
\operatorname{Var}(E(Y \mid X=x))=E\left([E(Y \mid X=x)-E(Y)]^{2}\right) .
$$

From this variance, the correlation ratio of $Y$ on $X$ is defined as (Kruskal, 1958)

$$
\eta(Y, X)=\left[\frac{\operatorname{Var}(E(Y \mid X=x))}{\operatorname{Var}(Y)}\right]^{1 / 2}=\frac{\sigma(E(Y \mid X=x))}{\sigma(Y)},
$$

where $\sigma(\ldots)$ indicates the standard deviation. Notice that $0 \leq \eta(Y, X) \leq 1$ whereas $-1 \leq \rho(X, Y) \leq 1$ Kruskal, 1958, pp. 816-817). As $\rho(X, Y)$ is a normalized 
$\operatorname{COV}(X, Y), \eta(Y, X)$ is a normalized $\operatorname{Var}(E(Y \mid X=x))$. Interestingly, the correlation ratio satisfies the following properties (Kruskal, 1958, pp. 816-817):

- $\eta(Y, X)=1$ if and only if $Y$ is a perfect function of $X$.

- $|\rho(X, Y)| \leq \eta(Y, X)$ with equality if and only if $Y$ is a linear function of $X$.

- $0 \leq \eta(Y, X) \leq 1$ (whereas $-1 \leq \rho(X, Y) \leq 1$ ). As $\rho(X, Y)$ is a normalized $\operatorname{COV}(X, Y), \eta(Y, X)$ is a normalized $\operatorname{Var}(E(Y \mid X=x))$.

It is well-known that (Kolmogorov, 1956, Poirier, 1995):

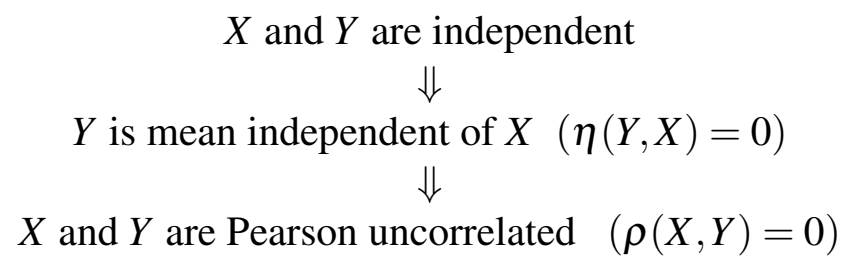

Proofs of the top to bottom implications have been provided by, e.g., Kolmogorov, 1956, pp. 60) or (Poirier, 1995, pp. 67). Mean independence implies Pearson uncorrelation but the converse (Pearson uncorrelation implies mean independence) is not necessarily true. To see it consider that

$$
p(X=x, Y=y)=\left\{\begin{array}{cc}
1 / 2 & \text { if } x=0 \text { and } y=-1 \\
1 / 4 & \text { if } x=-1 \text { and } y=1 \\
1 / 4 & \text { if } x=1 \text { and } y=1
\end{array}\right.
$$

Thus $E(X)=E(Y)=0$ and $E(X Y)=(1 / 4)(-1)+(1 / 2) 0+(1 / 4) 1=0$. Therefore $\operatorname{COV}(X, Y)=0$ but $Y$ is not mean independent of $X$ because $E(Y \mid X=-1)=1 \neq$ $E(Y \mid X=0)=-1$. Similarly, independence implies mean independence but the converse (mean independence implies independence) is not necessarily true (see Ferrer-i-Cancho et al. (2013a) for a counterexample).

In next section it will be shown that the correlation ratio is indeed more powerful than a correlation coefficient for testing whether Eq. 2 holds.

\section{How to test that $Z=a / X$}

\subsection{Non-parametric tests}

Here we consider three sample correlation statistics: the Pearson correlation $\rho(X, Y)$, the Spearman correlation $\rho_{S}(X, Y)$ and the correlation ratio $\eta(X, Y)$, and evaluate if 
they are significantly different from zero using a permutation test (a particular case of randomization test (Sokal and Rohlf, 1995, pp. 803-819)). In a sample of size $N$, $X$ and $Y$ can be seen as vectors, i.e. $X=\left\{x_{1}, \ldots, x_{i}, \ldots, x_{N}\right\}$ and $Y=\left\{y_{1}, \ldots, y_{i}, \ldots, y_{N}\right\}$, being $\left(x_{i}, y_{i}\right)$ the information associated to the $i$-th element of the sample. The $p$-value of $\rho(X, Y)$ is the probability that $X^{\prime}$, a random permutation of $X$, yields $\left|\rho\left(X^{\prime}, Y\right)\right| \geq|\rho(X, Y)|$. The test is two-sided as both positive and negative values of the sample $\rho(X, Y)$ that are significantly different from 0 are indicative of non-zero correlation (both $\rho(X, Y)=1$ and $\rho(X, Y)=-1$ are indicative of maximum correlation). The $p$-value of $\rho_{S}(X, Y)$ is calculated as that of $\rho(X, Y)$. The $p$-value of $\eta(Y, X)$ is the probability that $X^{\prime}$ (a random permutation of $X$ ) yields $\eta\left(Y, X^{\prime}\right) \geq \eta(Y, X)$. The test is one-sided as only positive values of the sample $\eta(X, Y)$ that are significantly large are indicative of non-zero correlation $(\eta(X, Y)$ is indicative of maximum correlation only when $\eta(X, Y)=1)$. The $p$-values were estimated with a Monte Carlo method generating $R=10^{7}$ uniformly random permutations. In the present article, we adopt a significance level of 0.05 .

For the analyses of this section, we used the same dataset of recent research (Hernández-Fernández et al., 2011, Baixeries et al., 2012, 2013, Ferrer-i-Cancho et al., 2013b). A summary of the statistical properties of the dataset is provided in Table 1. See Ferrer-i-Cancho and Forns (2009), Hernández-Fernández et al. (2011) for further details about the dataset. As the fact that $Y$ is mean independent of $X$ implies Pearson uncorrelation, i.e. $\operatorname{COV}(X, Y)=0$ (or $\rho(X, Y)=0$ ), Eq. 2 can be tested by means of the following procedure: if $\operatorname{COV}(X, Y)$ (or $\rho(X, Y)=0)$ is significantly different from 0 then reject Eq. 2, otherwise accept it. That procedure can be used to reject Eq. 2 in genomes, being $Z$ the mean chromosome length in bases of a species and $X$ being the number of chromosomes of that species (HernándezFernández et al., 2011).

Table 2 summarizes the results of the analysis of the Pearson correlation between $X$ (chromosome number) and $Y$ (genome size in bases) in genomes. A significant correlation is found in six out of eleven taxonomic groups. The test is conservative as it rejects Eq. 2 indirectly by means of a necessary condition for this equation to hold: $\operatorname{COV}(X, Y)=0$. Therefore, the five groups where the Eq. 2 could not be rejected might be false negatives. Pearson correlation is a measure of linearity between variables and has difficulties for capturing non-linear dependencies. A possible improvement is using a more powerful correlation metric such as $\rho_{S}(X, Y)$, the Spearman rank correlation coefficient, which is a measure of monotonic (linear or non-linear) dependency (Zhou, Tuncali, and Silverman, 2003). The Spearman rank correlation is a Pearson correlation on a rank transformation of the original values (Conover, 1999). The Spearman rank correlation test revealed that the majority of taxonomic groups (nine out of eleven) exhibit a significant correlation between $X$ and $Y$ that is incompatible with Eq. 22(Table 2). The exceptions are 
Table 1: A summary of the statistical properties of each taxonomic group. $N$ is the number of species. The mean $(\mu)$ and sample standard deviation $(\sigma)$ are provided for three variables: chromosome number $(X)$, genome size $(Y)$ in Million base pairs $(\mathrm{Mb})$ and mean chromosome size $(Z)$ in $\mathrm{Mb}$ per chromosome. The genome size in $\mathrm{Mb}$ is measured using the DNA $C$-value $(1 C)$. Accordingly, the number of chromosomes is measured using $1 n$. Mean and standard deviation were rounded to leave just one decimal digit.

\begin{tabular}{|c|c|c|c|c|c|c|c|}
\hline \multirow[b]{2}{*}{$\begin{array}{l}\text { Taxonomic } \\
\text { group }\end{array}$} & \multirow[b]{2}{*}{$N$} & \multicolumn{2}{|c|}{$X$} & \multicolumn{2}{|c|}{$Y$} & \multicolumn{2}{|c|}{$Z$} \\
\hline & & $\mu$ & $\sigma$ & $\mu$ & $\sigma$ & $\mu$ & $\sigma$ \\
\hline Fungi & 56 & 9.4 & 4.2 & 33.3 & 23.7 & 3.8 & 1.9 \\
\hline Angiosperms & 4706 & 14.2 & 9.6 & 5474.3 & 8902.5 & 515 & 847 \\
\hline Gymnosperms & 170 & 12 & 2.3 & 17695.4 & 7436.9 & 1489.6 & 613.3 \\
\hline Insects & 269 & 10.6 & 5 & 1240 & 1908.7 & 146 & 204.3 \\
\hline Reptiles & 170 & 19.1 & 3.8 & 2364.2 & 742.6 & 126 & 39.9 \\
\hline Birds & 99 & 38.8 & 31.8 & 1400.7 & 175 & 159.9 & 243.1 \\
\hline Mammals & 371 & 23.3 & 8.8 & 3413.2 & 822.1 & 182.1 & 134.2 \\
\hline $\begin{array}{l}\text { Cartilaginous } \\
\text { fishes }\end{array}$ & 52 & 36.7 & 8.2 & 5787.9 & 3100.4 & 166.5 & 95.1 \\
\hline Jawless fishes & 13 & 44 & 33.7 & 2442.7 & 1090.4 & 140.7 & 174.7 \\
\hline $\begin{array}{l}\text { Ray-finned } \\
\text { fishes }\end{array}$ & 647 & 27.6 & 10.7 & 1360.7 & 716.9 & 50.7 & 27.7 \\
\hline Amphibians & 315 & 14.2 & 5.5 & 18029 & 19952.3 & 1285.3 & 1373.4 \\
\hline
\end{tabular}


Table 2: Analysis of the correlation between $Y$ (genome size in bases) and $X$ (chromosome number). $D(X)$ is the proportion of different values of $X . \rho(X, Y)$ is the sample Pearson correlation coefficient, $\rho_{S}(X, Y)$ is the sample Spearman correlation coefficient and $\eta(Y, X)$ is the sample correlation ratio of $Y$ on $X$. The sample size corresponds to $N$ in Table 1 . The $p$-values were estimated using a Monte Carlo permutation test. The correlation statistics and the corresponding $p$-values were rounded to leave only two significant digits.

\begin{tabular}{llllllll}
\hline $\begin{array}{l}\text { Taxonomic } \\
\text { group }\end{array}$ & $D(X)$ & $\rho(X, Y)$ & $p$-value & $\rho_{S}(X, Y)$ & $p$-value & $\eta(Y, X)$ & $p$-value \\
\hline Fungi & 0.25 & 0.41 & 0.002 & 0.28 & 0.037 & 0.74 & 0.026 \\
Angiosperms & 0.02 & -0.0024 & 0.87 & -0.039 & 0.008 & 0.27 & 0.0013 \\
Gymnosperms & 0.08 & 0.1 & 0.18 & 0.32 & $2.9 \cdot 10^{-5}$ & 0.74 & $<10^{-7}$ \\
Insects & 0.08 & 0.09 & 0.14 & 0.22 & 0.0003 & 0.46 & 0.017 \\
Reptiles & 0.11 & 0.31 & $3.6 \cdot 10^{-5}$ & 0.24 & 0.0014 & 0.48 & 0.0031 \\
Birds & 0.36 & -0.029 & 0.78 & -0.033 & 0.74 & 0.78 & 0.0012 \\
Mammals & 0.11 & 0.3 & $<10^{-7}$ & 0.3 & $<10^{-7}$ & 0.68 & $<10^{-7}$ \\
Cartilaginous & 0.44 & 0.014 & 0.92 & -0.13 & 0.36 & 0.76 & 0.11 \\
fishes & & & & & & & \\
Jawless fishes & 0.77 & -0.76 & 0.0033 & -0.74 & 0.0049 & 0.98 & 0.054 \\
Ray-finned & 0.07 & 0.47 & $<10^{-7}$ & 0.49 & $<10^{-7}$ & 0.69 & $<10^{-7}$ \\
fishes & & & & & & & \\
Amphibians & 0.07 & 0.13 & 0.02 & 0.45 & $<10^{-7}$ & 0.58 & $<10^{-7}$ \\
\hline
\end{tabular}

birds and cartilaginous fishes. These findings confirm qualitatively the previous results with similar methods (Hernández-Fernández et al., 2011). Interestingly, there is a more powerful way of testing Eq. 2. testing directly if $Y$ is mean independent of $X$ from its definition, i.e. $E(Y \mid X=x)=E(Y)$ for any $x$, which is equivalent to $\eta(X, Y)=0$ (Kruskal, 1958). $\eta(Y, X)$ was not significantly large in two taxonomic groups: cartilaginous fishes and jawless fishes but the case of jawless fishes is border line: the estimated $\mathrm{p}$-value is 0.054 . The fact that this is the group with the smallest sample $(N=13)$, suggests that this might be a case of type II error.

Fig. 1 and Fig. 2 show histograms of the value of three correlation statistics in randomizations of the original data. As expected, the distribution for the Pearson and Spearman correlation statistics is centered at zero and symmetric. Fig. 22reveals crucial information to understand the taxonomic groups where the correlation ratio fails to reject the null hypothesis: the density of probability is concentrated on high 
values of the statistic. The case of jawless fishes is particularly intriguing: why the null hypothesis cannot be rejected when the correlation ratio is almost maximum? Table 2 shows that $\eta(Y, X)=0.98$ for jawless fishes while the maximum is $\eta(Y, X)=1)$. We believe that there is not a biological but a statistical reason for that: the test based on $\eta(Y, X)$ loses power (capacity to reject the null hypothesis) when the diversity of values of $X$ is high. If $\phi(X)$ is defined as the set of values of $x_{i}$ in the sample of a taxonomic group, i.e.

$$
\phi(X)=\cup_{i=1}^{N}\left\{x_{i}\right\}
$$

then one can define a simple diversity index $D(X)$ as the proportion of different values of $X$, i.e.

$$
D(X)=\frac{|\phi(X)|}{N} .
$$

Interestingly, the two groups with the largest $D(X)$ coincide with the two groups where the correlation ratio test fails to reject the null hypothesis (Table 2). It will shown that $\eta(Y, X)$ is maximum, i.e. $\eta(Y, X)=1$, when the diversity of $X$ is maximum, i.e. $D(X)=1$, regardless of the values of $Y$. Let us define $\phi(Y \mid w)$ as the set of values of $y_{i}$ such that $x_{i}=w$, i.e.

$$
\phi(Y \mid w)=\cup_{x_{i}=w, 1 \leq i \leq N}\left\{y_{i}\right\}
$$

and use $\operatorname{Var}(\ldots)$ to refer (in this context) to the sample variance and $\mu(Y \mid w)$ as the mean of $Y$ conditioned on $w\left(\mu(Y \mid w)\right.$ is the mean value of $y_{i}$ for $\left(x_{i}, y_{i}\right)$ pairs such that $x_{i}=w$ ). The sample correlation ratio can be defined as function of means and sample variances as

$$
\eta(Y, X)=\left(\frac{\operatorname{Var}(\mu(Y \mid x))}{\operatorname{Var}(Y)}\right)^{1 / 2}
$$

If $D(X)=1$, i.e. $|\phi(X)|=N$ (Eq. 11), then $|\phi(Y \mid x)|=1$ for every $x$ in $\phi(X)$. This has a very important consequence, namely that $\mu\left(Y \mid x_{i}\right)=y_{i}$, which yields $\operatorname{Var}(\mu(Y \mid x))=\operatorname{Var}(Y)$ and therefore $\eta(Y, X)=1$.

The fact that $\eta(Y, X)=1$ when there are not repeated values of $X$ means that $\eta(Y, X)$ will keep being maximum for any randomization of the data and thus the p-value of $\eta(Y, X)$ is 1 . Put differently, the data might be showing a perfect correlation between $X$ and $Y$ but the absence of repeated values of $X$ can lead to a type II error (false negative) when $D(X)=1$. We believe that this can also happen when $D(X)<1$ but $D(X)$ is sufficiently large. Not surprisingly, the only two groups where the correlation ratio test fails to reject the null hypothesis at a significance level of 0.05 , i.e. cartilaginous fishes and jawless fishes, are the two groups with the largest $D(X)(D(X)=0.44$ and $D(X)=0.77$, respectively; Table 2). $D(X)$ 
allows one to solve the puzzle of why the density of probability of the correlation ratio is concentrated on high values of $\eta(Y, X)$, specially for cartilaginous fishes and jawless fishes (Fig. 2): their high value of $D(X)$ is biasing $\eta(Y, X)$ towards high values in randomization of the original data, no matter how the values of $X$ and $Y$ combine under the null hypothesis.

\subsection{A parametric test}

The hypothesis of $Z=a / X$ has been accepted with the only support that the fit of $Z=a X^{b}$ yields $b \approx-1$ (Solé, 2010). This procedure is very prone to type II error (accepting a false null hypothesis) as it needs that $Z=a X^{b}$ holds first (Baixeries et al., 2013, Ferrer-i-Cancho et al., 2013b). Our analysis shows that for $Z=a / X$ to hold, it is not only necessary that $b \approx-1$ is retrieved but also $a \approx \mu(Y)$, where $\mu(Y)$ is the mean of $Y$, an estimate of $E(Y)$ (recall Theorem 2.1, see also (Ferrer-iCancho et al., 2013a)). Even if $a \approx \mu(Y)$ and $b \approx-1$, type II errors are not excluded and minimizing them needs evidence that $Z=a X^{b}$ is well-supported by data.

\section{Discussion}

We have argued that claiming that $Z$ scales with $X$ following a very particular form of Menzerath-Altmann law, i.e. Eq. 2, is indeed equivalent to claiming that $E(Z \mid X)=E(Y) / X$, which is indeed equivalent to claiming that $Y$ is mean independent of $X$. We have also presented a new correlation ratio test revealing that Eq. 2 could only hold in cartilaginous fishes and maybe also in jawless fishes. The two major conclusion of our article are that

- The trivial scaling defined by Eq. 2 is the exception, not the rule.

- The correlation ratio is at least one of the correlation statistics to consider for testing the null hypothesis that $Z=a / X$.

While the Pearson correlation test rejects Eq. 2 in 6 taxonomic groups, the Spearman correlation and the correlation ratio tests do it in 9 taxonomic groups (Table 2). The two most powerful tests might be failing because of type II errors. This possibility is apparently easier to defend for the correlation ratio from the perspective of insufficient sampling: the correlation ratio test fails to reject the null hypothesis exactly for the two groups having the smallest sample size (jawless fishes and cartilaginous fishes). In contrast, the Spearman correlation test fails with the second and fourth taxonomic groups with the smallest sample size, i.e. jawless fishes and 

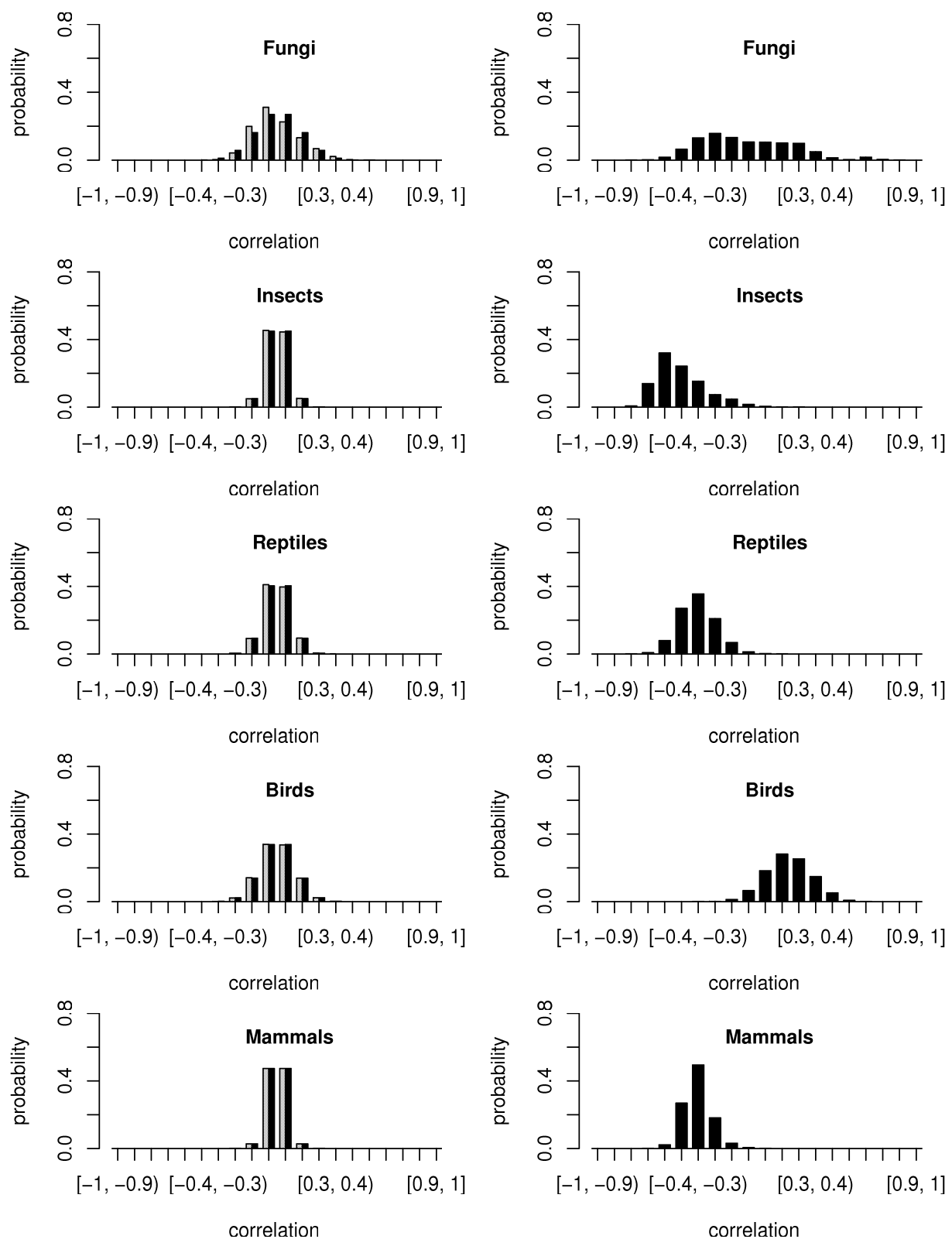

Figure 1: Normalized histograms of the correlations statistics. The range of variation of every statistics is split into 20 bins. Left column: histograms of the Pearson (black) and Spearman (gray) rank correlation with a bin size of 0.1. Right column: histogram of the correlation ratio with a bin size of $0.05 .10^{7}$ randomizations were used. 
birds (recall Table 1). A deeper explanation of the failure of the correlation ratio is offered by $D(X)$.

We have shown that the power of the correlation test depends on the diversity of values of $X$, losing power completely if that diversity is maximum. The two groups where that statistic fails to reject the null hypothesis are not only the two groups with maximum $D(X)$ but also the two groups with the smallest $N$ (Table 1 and 2). The latter suggest that the true reason for the failure is that the samples of these two groups are not large enough for significance to emerge. Indeed, $D(X)$ is a function of $N$. However, if a small sample size was the only reason for the failure to reject the null hypothesis, one would also expect that fungi (with $N=56$ ) were also problematic, but their p-values are about half the significance level of 0.05 . While the value of $N$ cartilaginous fishes and fungi are very similar (the value of $N$ of cartilaginous fishes is about $92 \%$ of the value of $N$ of fungi; Table 11), the differences between both groups in terms of $D(X)$ are much larger (the value of $D(X)$ of cartilaginous fishes is about $176 \%$ the value of $D(X)$ of fungi; Table 2). Therefore, a high $D(X)$ is more likely to be the primary reason for the failures of the correlation ratio tests (notice that $N$ could be very large but the sample $\eta(Y, X)$ may not be significantly large because $D(X)=1$ ).

Whether the correlation ratio is a more powerful statistic than the Spearman rank correlation must be investigated further. If the significant level is raised to 0.01 , then it turns out that the Spearman rank correlation allows one to reject the null hypothesis in 8 taxonomic groups while the correlation ratio does it in 7 groups (Table 2). However, the difference is small and both statistics may need to be used jointly.

The fact that $Z=a / X$ does not hold in many taxonomic groups must be interpreted with care. The taxonomic groups are not totally independent and thus the number of groups in which a statistic rejects the null hypothesis might be uninformative: it may simply reflect the existence of a common ancestor in their evolutionary history (the same argument could be applied to the groups where the null hypothesis could not be rejected: their number might be uninformative). However, the hypothesis of a common ancestor is incomplete at present because

- A demonstration of the existence of such ancestor is necessary. That demonstration would require a careful analysis of the transmission of traits throughout phylogeny (e.g., Dunn, Greenhill, Levinson, and Gray (2011)) and this is beyond the scope of this article.

- An explanation of why the common ancestor group adopted that property would still be required. 
However, the many rejections of the null hypothesis might be due to the convergence of distinct taxonomic groups to the same statistical pattern by some general constraints, maybe physical or biological principles. This alternative to the common ancestor is supported by our suspect that the reason why the correlation ratio has failed in two groups are not biological but rather statistical (type II errors). The possibility that one of those general constraints is the viability and well-formedness of chromosomes is reviewed next.

The random breakage model where $X$ and $Y$ are independent and uniformly distributed (Solé, 2010) fails to fit the majority of taxonomic groups because independence is a particular case of mean independence (Ferrer-i-Cancho et al., 2013a) and mean independence fails in at least nine out of eleven taxonomic groups (Table 22. Interestingly, it has been argued that independence between $X$ and $Y$ is problematic as it can lead to organisms with empty chromosomes or empty chromosome parts (Baixeries et al. 2012). Notice that $Y$ does not need to be the size of genome in bases. It could be the size in units between the base and the chromosome.

The problem of empty components also concerns mean independence. The condition for not expecting empty chromosomes for a given $x$ (a concrete value of $X)$ is

$$
\begin{aligned}
E(Z \mid X=x) & \geq 1 \\
\frac{1}{x} E(Y \mid X=x) & \geq 1 .
\end{aligned}
$$

For that $x$, the condition in Eq. 14 becomes $E(Y) \geq x$ when $Y$ is mean independent of $X$ as $E(Y \mid X=x)=E(Y)$ in that case. Thus, under mean independence, empty chromosomes are expected in an organism of $x$ chromosomes if $E(Y)<x$. Notice that expecting that Eq. 14 holds on average for any $x$, leads to

$$
\begin{aligned}
E(E(Y \mid X=x)) & \geq E(X) \\
E(Y) & \geq E(X)
\end{aligned}
$$

thanks to the law of total probability for expectations (DeGroot and Schervish, 2012, pp. 258). The restrictions defined by Eqs. 14 and 15 are perhaps very simple but Baixeries et al. (2012) considered more elaborated constraints for the viability of an organism based upon the parts making an ideal chromosome: a centromere, two telomeres and a couple of intermediate regions. Those viability constraints lead to a deviation from Menzerath-Altmann law with $b=-1$ and $c=0$ (see Fig. 3 of Baixeries et al.(2012)), which Theorem 2.1 allows one to interpret unequivocally as a departure from mean independence. Therefore, the viability and well-formedness of chromosomes is not compatible with mean independence either. This suggests that a common ancestor might not be the only reason for the recurrent failure of 
$Z=a / X$ in taxonomic groups. Indeed, the existence of general and lineage independent constraints is a more parsimonious hypothesis that has to be investigated further.

The negative Spearman rank correlation between $Z$ and $X$, known as Menzerath's law, defies a trivial explanation in genomes (Ferrer-i-Cancho and Forns, 2009. Wilde and Schwibbe, 1989). Claiming that the scaling defined by Eq. 2 is inevitable (Solé, 2010) is equivalent to claiming that $Y$ must be mean independent of $X$ in any circumstance, a very strong requirement for real language, music and genomes. Eq. 2 should be regarded as a baseline instead of a fact for the relationship between chromosome number and mean chromosome length. Rather than signs of complexity, certain power laws (the value of the exponent can be crucial) might indicate the control that must be considered before any claim of "sufficient biological complexity" can be made.

\section{Acknowledgements}

This article has benefited enormously from the comments of anonymous reviewers. We are grateful to P. Delicado, R. Gavaldà and E. Pons for their valuable mathematical insights. We owe the counterexample showing that uncorrelation does not imply mean independence to P. Delicado. We are also grateful to G. Bel-Enguix and N. Forns for helpful discussions. This work was supported by the grant Iniciació $i$ reincorporació a la recerca from the Universitat Politècnica de Catalunya, the grants BASMATI (TIN2011-27479-C04-03) and OpenMT-2 (TIN2009-14675C03) from the Spanish Ministry of Science and Innovation and the grant 2/0038/12 from the VEGA funding agency (JM).

\section{References}

Altmann, G. (1980): "Prolegomena to Menzerath's law," Glottometrika 2, 2, 1-10.

Baixeries, J., A. Hernández-Fernández, and R. Ferrer-i-Cancho (2012): "Random models of Menzerath-Altmann law in genomes," Biosystems, 107, 167-173.

Baixeries, J., A. Hernández-Fernández, N. Forns, and R. Ferrer-i-Cancho (2013): "The parameters of Menzerath-Altmann law in genomes," Journal of Quantitative Linguistics, 20, 94-104.

Boroda, M. G. and G. Altmann (1991): "Menzerath's law in musical texts," Musikometrica, 3, 1-13.

Cameron, A. C. and P. K. Trivedi (2009): Microeconometrics: Methods and Applications, Cambridge: Cambridge University Press. 
Conover, W. J. (1999): Practical nonparametric statistics, New York: Wiley, 3rd edition.

Cramer, I. M. (2005): “The parameters of the Altmann-Menzerath law," Journal of Quantitative Linguistics, 12, 41-52.

Crathorne, A. R. (1922): "Calculation of the correlation ratio," Journal of the American Statistical Association, 18, 394-396.

DeGroot, M. H. and M. J. Schervish (2012): Probability and statistics, Boston: Wiley, 4th edition.

Dunn, M., S. J. Greenhill, S. C. Levinson, and R. D. Gray (2011): "Evolved structure of language shows lineage-specific trends in word-order universals," Nature, 473, 79-82.

Ferrer-i-Cancho, R., J. Baixeries, and A. Hernández-Fernández (2013a): "Erratum to "Random models of Menzerath-Altmann law in genomes" (BioSystems 107 (3), 167-173)," Biosystems, 111, 216-217.

Ferrer-i-Cancho, R. and N. Forns (2009): "The self-organization of genomes," Complexity, 15, 34-36.

Ferrer-i-Cancho, R., N. Forns, A. Hernández-Fernández, G. Bel-Enguix, and J. Baixeries (2013b): "The challenges of statistical patterns of language: the case of Menzerath's law in genomes," Complexity, 18, 11-17.

Hernández-Fernández, A., J. Baixeries, N. Forns, and R. Ferrer-i-Cancho (2011): "Size of the whole versus number of parts in genomes," Entropy, 13, 1465-1480.

Khanin, R. and E. Wit (2006): "How scale-free are biological networks," Journal of Computational Biology, 13, 810-818.

Kolmogorov, A. N. (1956): Foundations of the theory of probability, New York: Chelsea Publising Company, 2nd edition.

Kruskal, W. H. (1958): "Ordinal measures of association," Journal of the American Statistical Association, 53, 814-861.

Li, W. (1992): "Random texts exhibit Zipf's-law-like word frequency distribution," IEEE T. Inform. Theory, 38, 1842-1845.

Li, W. (2012): "Menzerath's law at the gene-exon level in the human genome," Complexity, 17, 49-53.

May, R. M. and M. P. H. Stumpf (2000): "Species-area relations in tropical forests," Science, 290, 2084-2086.

McCowan, B., L. R. Doyle, J. M. Jenkins, and S. F. Hanser (2005): "The appropriate use of Zipf's law in animal communication studies," Anim. Behav., 69, F1-F7.

Menzerath, P. (1954): Die Architektonik des deutschen Wortschatzes, Bonn: Dümmler.

Miller, G. A. (1968): “Introduction," in The Psycho-Biology of Language: an Introduction to Dynamic Psychology (by G. K. Zipf), Cambridge, MA, USA: MIT 
Press, $\mathrm{v}-\mathrm{X}$.

Miller, G. A. and N. Chomsky (1963): "Finitary models of language users," in R. D. Luce, R. Bush, and E. Galanter, eds., Handbook of Mathematical Psychology, volume 2, New York: Wiley, 419-491.

Poirier, D. J. (1995): Intermediate Statistics and Econometrics: A Comparative Approach, Cambridge: MIT Press.

Ritz, C. and J. C. Streibig (2008): Nonlinear regression with R, New York: Springer.

Sokal, R. R. and F. J. Rohlf (1995): Biometry. The principles and practice of statistics in biological research, New York: W. H. Freeman and Co., 3rd edition.

Solé, R. V. (2010): “Genome size, self-organization and DNA's dark matter,' Complexity, 16, 20-23.

Stumpf, M., P. Ingram, I. Nouvel, and C. Wiuf (2005): "Statistical model selection methods applied to biological network data," Trans. Comp. Syst. Biol., 3, 65âĂŞ77.

Stumpf, M. P. H. and P. J. Ingram (2005): "Probability models for degree distributions of protein interaction networks," Europhysics Letters, 71, 152.

Stumpf, M. P. H. and M. A. Porter (2012): "Critical truths about power laws," Science, 335, 665-666.

Suzuki, R., P. L. Tyack, and J. Buck (2005): “The use of Zipf's law in animal communication analysis," Anim. Behav., 69, 9-17.

Tanaka, R., T.-M. Yi, and J. Doyle (2005): "Some protein interaction data do not exhibit power law statistics," \{FEBS\} Letters, 579, 5140 - 5144.

Teupenhayn, R. and G. Altmann (1984): “Clause length and Menzerath's law." Glottometrika, 6, 127-138.

Tjørve, E. (2003): "Shapes and functions of species-area curves: a review of possible models," Journal of Biogeography, 30, 823-832.

Wilde, J. and H. Schwibbe (1989): "Organizationsformen von Erbinformation Im Hinblick auf die Menzerathsche Regel," in G. Altmann and M. H. Schwibbe, eds., Das Menzerathsche Gesetz in informationsverarbeitenden Systemen, Hildesheim: Olms, 92-107.

Wooldridge, J. M. (2010): Econometric Analysis of Cross Section and Panel Data, Cambridge: MIT Press.

Zhou, K., K. Tuncali, and S. G. Silverman (2003): “Correlation and simpler linear regression," Radiology, 227, 617-628. 

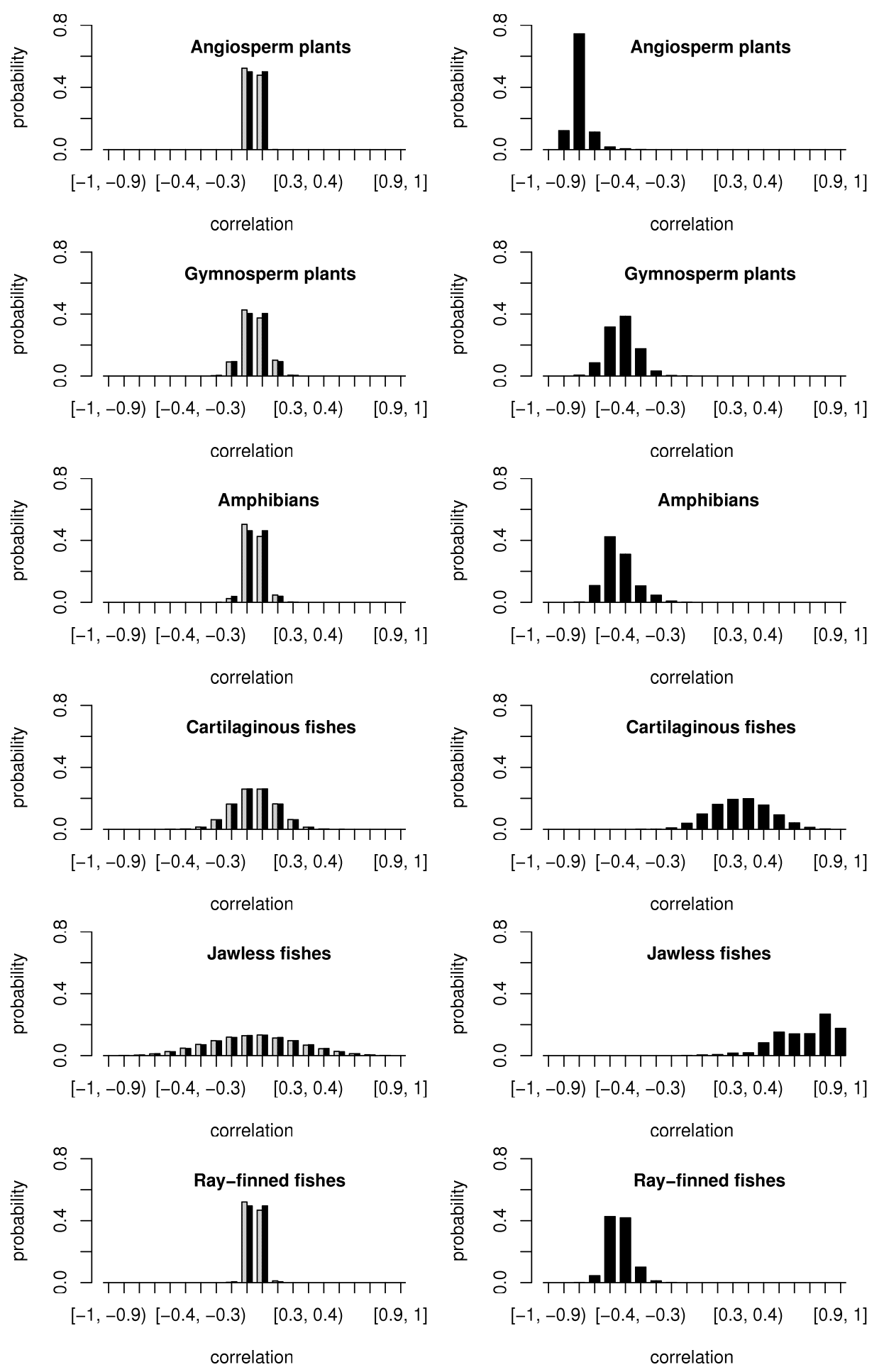

Figure 2: The same as in Table 1 for other taxonomic groups. 